\title{
Fast Growing
}

National Cancer Institute

\section{Source}

National Cancer Institute. Fast Growing. NCI Thesaurus. Code C124825.

An indication that something is growing at a fast rate. 